\title{
Experimental Study on Silicon Nanocrystals Rich Lanthanum Fluoride Films for Future Electronic Devices
}

\author{
Md. Ferdous Rahman ${ }^{1 *}$, Sheikh Rashel Al Ahmed ${ }^{2}$, Golam Saklayen ${ }^{3}$ and \\ Abu Bakar Md. Ismail ${ }^{3}$ \\ ${ }^{1}$ Department of Electronics and Telecommunication Engineering, Begum Rokeya \\ University, Rangpur, Bangladesh. \\ ${ }^{2}$ Department of Electronics and Telecommunication Engineering, Pabna Science \& \\ Technology University, Pabna, Bangladesh. \\ ${ }^{3}$ Department of Applied Physics and Electronic Engineering, University of Rajshahi, \\ Rajshahi 6205, Bangladesh.
}

*Corresponding author: ferdousapee@gmail.com

\begin{abstract}
Feasibility for the future electronic devices a thorough investigation on Silicon nanocrystals (Si-NCs) rich Lanthanum Fluoride (LaF3) film fabricated using a novel onestep chemical method has been reported here. Colloidal solution of Si-NCs in hydrofluoric acid (HF) was prepared from meso-porous silicon by ultrasonic vibration (sonication). On a silicon ( $\mathrm{Si}$ ) substrate $\mathrm{LaCl}_{3}$ solution in $\mathrm{HCL}$ is allowed to react with the colloidal solution of prepared Si-NCs. $\mathrm{LaCl}_{3}$ reacts with $\mathrm{HF}$ of $\mathrm{Si}-\mathrm{NCs}$ solution and produces $\mathrm{LaF}_{3}$ crystals that deposits on the silicon substrate as a film embedding Si-NCs. This is a novel single step chemical way of depositing $\mathrm{LaF}_{3}$ insulating layer embedding $\mathrm{Si}-\mathrm{NCs}\left(\mathrm{LaF}_{3}: \mathrm{Si}-\mathrm{NCs}\right)$. The XRD and EDX analysis of the deposited film show a polycrystalline and non-stoichiometric nature of $\mathrm{LaF}_{3}$. The presence of Si-NCs was confirmed by SEM. Application of this material has been tested for low-voltage operating non-volatile memory (NVM) and Schottky junction solar cells. The $\mathrm{Al} / \mathrm{LaF}_{3}: \mathrm{Si}-\mathrm{NCs} / \mathrm{Al}$ structure as NVM offered a memory window of $525 \mathrm{mV}$ at a programming and erasing bias of 2V. LaF3:Si-NCs films showed strong light absorption. Current-Voltage (I-V) characteristics of the Schottky device in $\mathrm{ITO} / \mathrm{LaF}_{3}: \mathrm{Si}-\mathrm{NCs} / \mathrm{Al}$ structure showed a dependency on the incident light intensity where current was varied in the range of $5 \mathrm{~mA}$ to $40 \mathrm{~mA}$ and under various light illumination i.e., 400 lux to 1200 lux. Experimental results show a lot of promise of Si-NCs-rich $\mathrm{LaF} 3$ film to be used as an insulating film in non-volatile memory as well as a photoactive material in Schottkey junction solar cell.
\end{abstract}

Keywords: Hybrid-solar-cell; Macroporous; Exciton; Bulk-heterojunction; Electrochemical-etching; Fill-factor; Conversion-efficiency.

\section{INTRODUCTION}

Recently researchers have been considering nanocrystal-based memory devices as a solution to ultra-large scale integration of electronic nonvolatile memories. One major barrier to such integration of NVMs is the local defect related leakage. Using discrete nanocrystals instead of the conventional continuous floating gate as charge storage nodes, local-defect-related leakage can be reduced efficiently to improve data retention [1]. In this regard, discrete-trap type semiconductor storage materials such as Si nanocrystals (Si-NCs) embedded in a dielectric matrix have been demonstrated as potential candidates for the fabrication of high-speed, high-density, low power-consuming, and nonvolatile memories [2-6]. Therefore, the poly-silicon-oxide-nitride-oxide-silicon (SONOS)-type 
structure memories including nanocrystal memories have recently attracted much attention for the application in the next-generation nonvolatile memories [7], [14] because of their great potential for achieving high program/erase (P/E) speed, low programming voltage and low power performance. For conventional SONOS, erase saturation and vertical stored charge migration [13-14] are the major drawbacks; while for nanocrystal memories good enough charge keeping capability of the discrete storage nodes and the formation of nanocrystals with constant size, high density and uniform distribution are the extremely challenging issues. $\mathrm{LaF}_{3}$ has been chosen as alternative candidates for gate insulator because of their large band gap, high dielectric constant, and large refractive index. Moreover, the lanthanide fluorides $\left(\mathrm{LaF}_{3}\right)$ show good characteristics without preformed interfacial layer, and regarded as high-k dielectrics [15]. In this work our goal has been to fabricate Si-NCs-rich $\mathrm{LaF}_{3}$ film to be used as an insulating film in non-volatile memory as well as a photoactive material in Schottky junction solar cell.

\section{EXPERIMENTAL}

Colloidal suspensions of silicon nanocrystals were fabricated from porous silicon in hydrofluoric (HF) acid by sonication (Ultrasonic Vibra cell, VCX 130) at a frequency of $20 \mathrm{kHz}$. Porous Silicon (PS) samples were prepared by standard electrochemical etching in a homemade double-tank cell [Figure-1] of p-type $<100>\mathrm{Si}$ wafers in a HF (48\%): Ethanol $=2.5: 1$ solution at different current densities for 30 minutes at room light temperature and illumination.

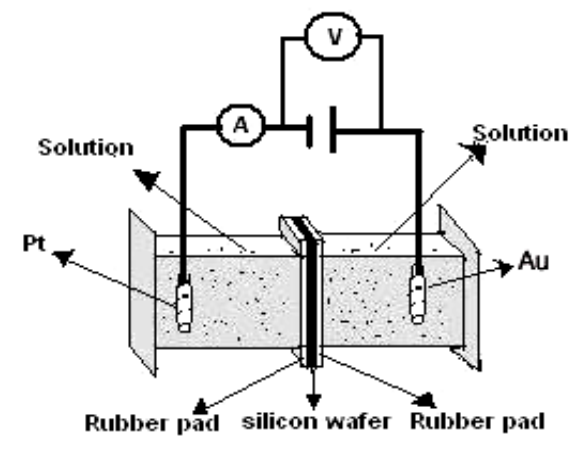

Fig-1(a): Complete double-tank cell setup for PS fabrication.

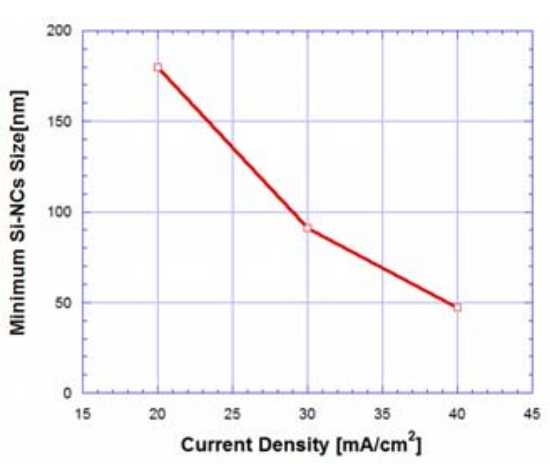

Fig-1(b) Variation of the minimum Si-NCs size with current densities

Just fabricated PS samples were readily immersed in hydrofluoric acid (HF) in a small plastic container and this container was immersed in water. Ultra sonic wave was applied to the water through the ultrasonic probe to vibrate the PS layer for about 60 minutes. The sonication was done at ultrasonic power 40 watt (amplitude $80 \%$ ). The sonication crumbles the top layer of the as made PS, a weakly interconnected nanostructures network into ultra-small particles and produces luminescent, reddish, colloidal suspensions of Si-NCs. The presence of silicon nanocrystal ( $\mathrm{Si}-\mathrm{NCs}$ ) was verified by scanning electron microscope (Models S-3400N, Hitachi). As shown in Fig-1(b) the size 
of the Si-NCs were dependent on the etching current density. Thus some sort of NC size tunability was obtained.

This colloidal solution of silicon nanocrystals in HF is allowed to react with Lanthanum chloride $\left(\mathrm{LaCl}_{3}\right)$ solution in hydrochloric acid $(\mathrm{HCl})$ at room temperature on the Aluminium coated glass substrate. The chemical reaction between the $\mathrm{LaCl}_{3}$ solution and colloidal solution of Si-NCs in HF can be given as

$$
\mathrm{LaCl}_{3}+\mathrm{HF} \rightarrow \mathrm{LaF}_{3}+\mathrm{HCl}
$$

The memory device was finalized by coating the top aluminium layer. For the fabrication of Schottky junction solar cell, the colloidal solution of $\mathrm{LaF}_{3}$ with Si-NCs were made acid-free by centrifuging process using ultra cooling Sigma Laboratory Centrifuges Machine (Sigma 3K30 Centrifuge). $\mathrm{LaF}_{3}$ with Si-NCs thin films were spin-cast onto indium tin oxide (ITO)-coated glass substrates at different rpm for different rotating times by VTC 100 Vacuum Spin Coater. Before applying colloidal solution of $\mathrm{LaF}_{3}$ with SiNCs on ITO coated flat glass substrate the spin coater VTC 100was programmed to spin with the specified parameters.

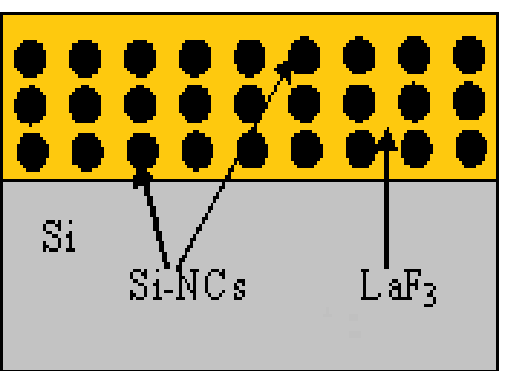

Fig - 2: Schematic diagram of a fabricated Non-volatile memory device

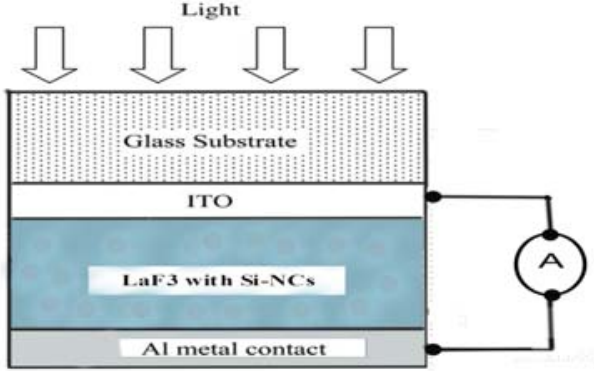

Fig - 3: Schematic diagram of a fabricated Schottky junction solar cell

For I-V characterization of the $\mathrm{LaF}_{3}$ with Si-NCs deposited on ITO coated glass sample, Aluminium (Al) film was deposited onto the $\mathrm{LaF}_{3}$ films with Si-NCs. Then the copper wires were connected onto the Al and ITO layer with silver paste. The arrangement for $\mathrm{I}-\mathrm{V}$ characterization is shown in [Fig-2].

\section{RESULTS AND DISCUSSION}

The X-ray diffraction of the deposited layer shows a polycrystalline $\mathrm{LaF}_{3}$ deposition on silicon. Scanning Electron Microscopy (SEM) has been used to detect silicon nanocrystals (Si-NCs) embedded within the $\mathrm{LaF}_{3}$ insulating layer and also investigated fabricated device of cross sectional view. EDX study on the $\mathrm{LaF}_{3}$ films with $\mathrm{Si}-\mathrm{NCs}$ confirmed the presence of $\mathrm{LaF}_{3}$ and $\mathrm{Si}$. As shown in fig-5 the EDX spectrum showed small carbon in the system because carbon tape were used for contact the sample with the sample stage. Table- 1 shows the atom and weight percentage of various elements in the 
$\mathrm{LaF}_{3} / \mathrm{Si}-\mathrm{NCs} / \mathrm{Si}$ system which confirms the non-stoichiometric nature of $\mathrm{LaF}_{3}$ deposited sample.
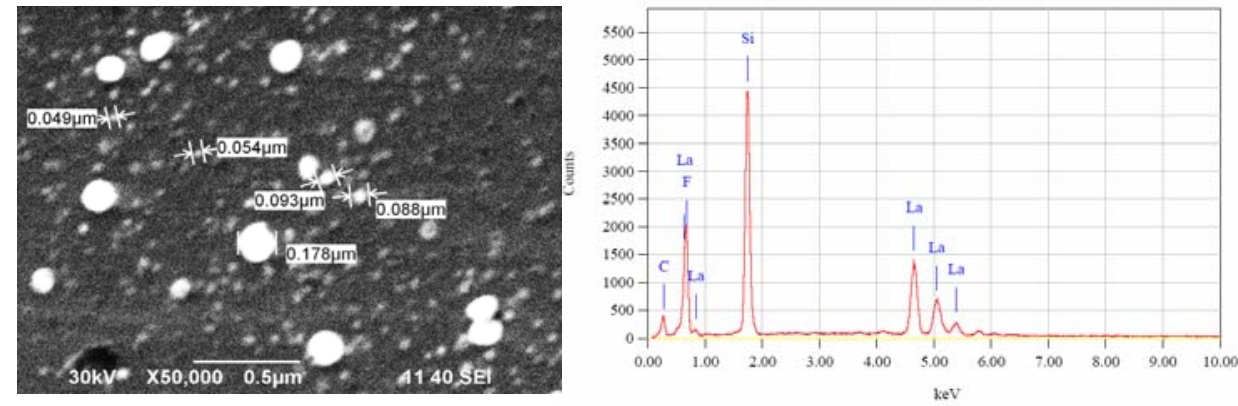

Fig-4: SEM on the $\mathrm{LaE}_{3}$ surface

Fig-5: EDX spectra of $\mathrm{LaF}_{3}$ films with Si-NCs

Table-1: Atom percentage of the whole system

\begin{tabular}{|l|l|l|}
\hline Elements & Weight\% & Atom\% \\
\hline $\mathrm{C}$ & 26.40 & 9.63 \\
\hline $\mathrm{F}$ & 21.49 & 25.55 \\
\hline $\mathrm{Si}$ & 25.48 & 20.49 \\
\hline $\mathrm{La}$ & 26.63 & 4.33 \\
\hline
\end{tabular}

The memory measurement has been performed by an impedance analyzer. Capacitancevoltage $(\mathrm{C}-\mathrm{V})$ study of the $\mathrm{MIS}\left[\mathrm{Al} / \mathrm{LaF}_{3}: \mathrm{Si}-\mathrm{NCs} / \mathrm{Al}\right]$ structure reveals that resonant tunneling of electron and charge storage was there when the MIS was biased from accumulation to inversion, which created a memory window. This type of memory window is called hysteresis. The MIS structure showed hysteresis for forward and reverse bias scan, enabling the structure to be used a non-volatile memory. The CapacitanceVoltage $(\mathrm{C}-\mathrm{V})$ curves observed of the $\mathrm{LaF}_{3}$ layer deposited nonvolatile memory device for various frequencies and various bias voltage and comparative these $(\mathrm{C}-\mathrm{V})$ curves.

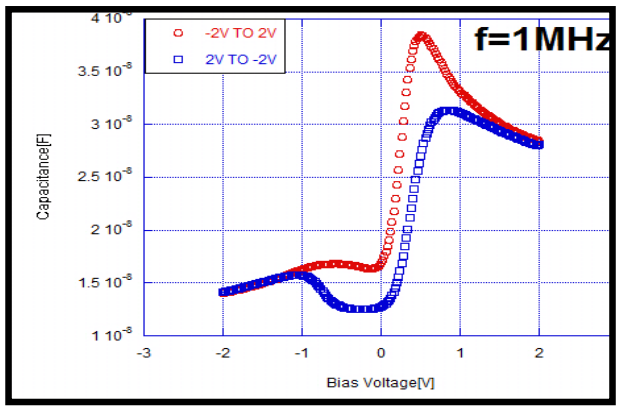

Fig-6: C-V response of $\mathrm{Al} / \mathrm{LaF}_{3}: \mathrm{Si}-\mathrm{NCs} / \mathrm{Al}$ 
The $\mathrm{C}-\mathrm{V}$ characteristics were measured over a voltage range $[-2 \mathrm{~V}$ to $2 \mathrm{~V}$ and back to $2 \mathrm{~V}],[-4 \mathrm{~V}$ to $4 \mathrm{~V}$ and back to $-4 \mathrm{~V}],[-6 \mathrm{~V}$ to $6 \mathrm{~V}$ and back to $-6 \mathrm{~V}]$ at a room temperature with frequencies $500 \mathrm{~Hz}, 1 \mathrm{MHz}, 2 \mathrm{MHz}, 3 \mathrm{MHz}, 4 \mathrm{MHz}$ and $5 \mathrm{MHz}$. At $1 \mathrm{MHz}$ frequency and over a voltage range [ $-2 \mathrm{~V}$ to $2 \mathrm{~V}$ and back to $-2 \mathrm{~V}$ ] hysteresis voltage difference (memory window) $(525 \mathrm{mV})$ is quite satisfactory [Fig-6]. It is clearly seen from fig-6 that a memory window of about $525 \mathrm{mV}$ is achievable at a bias voltage of $(-2 \mathrm{~V}$ to $+2 \mathrm{~V})$, indicating the huge promise of the structure to be used as a non-volatile memory device.

All experimental results of optical and electrical are discussed here for $120 \mathrm{~nm}$ of postannealed $\mathrm{LaF}_{3}$ films with Si-NCs. There were three $\left[\mathrm{LaF}_{3}: \mathrm{SiNCs} / \mathrm{ITO} / \mathrm{Glass}\right]$ structures fabricated for optical study and each had similar performance. The optical absorption spectrum of $\mathrm{LaF}_{3}$ films with and without Si-NCs on ITO coated glass substrate were measured and are presented in [Fig-7]. The $\mathrm{LaF}_{3}$ films with Si-NCs showed strong absorption.

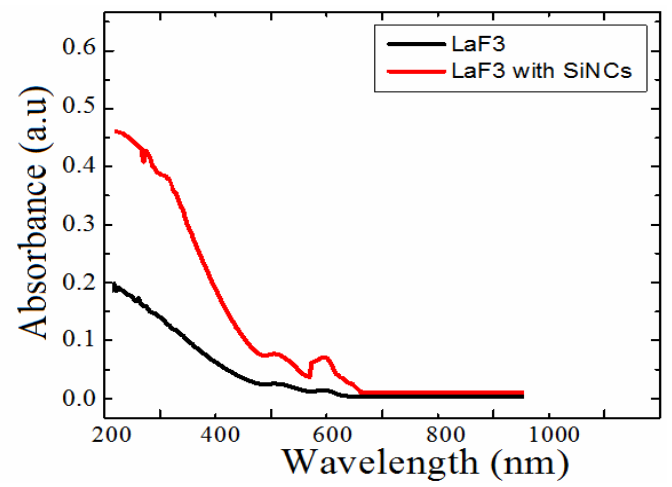

Fig-7: Absorption of $\mathrm{LaF}_{3}$ film with Si-NCs and $\mathrm{LaF}_{3}$ film.

The I-V characteristics of the film showed a dependency on the incident light intensity where current changed under various light illumination [Fig-8(a) \& 8(b)]. Experimental results shows a lot of promise of $\mathrm{Si}-\mathrm{NCs}$ embedded $\mathrm{LaF}_{3}$ layer to be used as an insulating layer in MIS devices as well as a photoactive material in Schottkey junction solar cell.

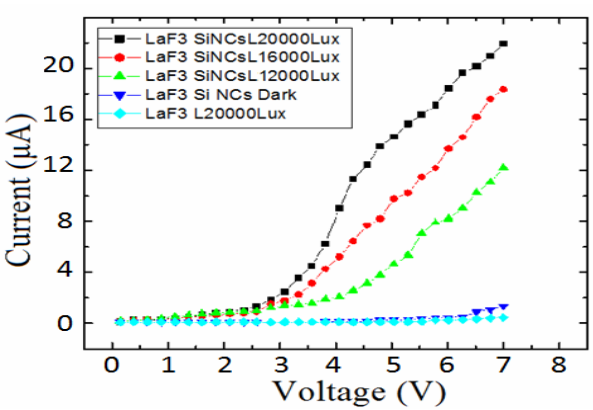

(a) Forward

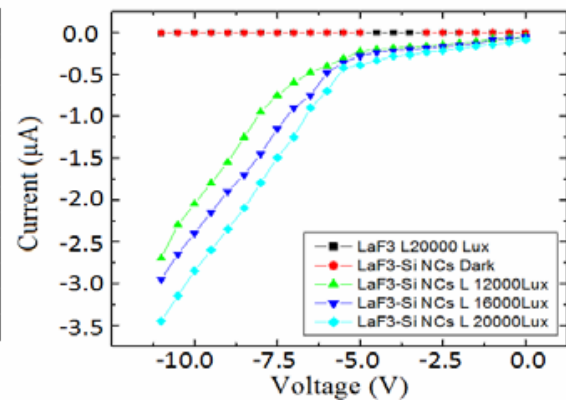

(b) Reverse 


\section{CONCLUSIONS}

Fig-8: Light dependent I-V response of ITO/ $\mathrm{LaF}_{3}: \mathrm{Si}-\mathrm{NCs} / \mathrm{Al}$

In this work, The $\mathrm{Al} / \mathrm{LaF}_{3}: \mathrm{Si}-\mathrm{NCs} / \mathrm{Al}$ structure was tested as NVM and a memory window of $525 \mathrm{mV}$ was obtained at a programming and erasing bias of $2 \mathrm{~V}$. The $\mathrm{LaF}_{3}$ : Si-NCs films showed strong absorption.I-V characteristics of ITO/LaF $/ \mathrm{Si} / \mathrm{Al}$ structure showed a dependency on the incident light intensity where current changed under various light illumination. Experimental results show a lot of promise of $\mathrm{Si}-\mathrm{NCs}-$ rich $\mathrm{LaF}_{3}$ film to be used as an insulating film in non-volatile memory as well as a photoactive material in Schottky junction solar cell. In future, Si-NCs can be used in the conventional Si-Solar cell to enhance the conversion efficiency of solar cell.

\section{Acknowledgment}

This work was funded by the Department of Applied Physics and Electronic Engineering, University of Rajshahi, Rajshahi 6205, Bangladesh. We acknowledge the help of Atomic Energy Centre, Dhaka for the SEM characterization.

\section{References}

[1] Aaron VY, Leburton JP: Flash memory: towards single-electronics. IEEE Potentials, pp.21:35, 2002.

[2] Tiwari S, Rana F, Hanafi H, Hartstein A, Crabbe EF, Chan K: A silicon nanocrystals based memory. Appl. Phys. Lett. , 68:1377. 1996.

[3] Hanafi HI, Tiwari S, Khan I: Fast and long retention-time nano-crystal memory. IEEE Trans Electron Devices, 43:1553, 1996.

[4] King YC, King TJ, Hu C: Charge-trap memory device fabricated by oxidation of Sil-x Gex. IEEE Trans Electron Devices, 48:696, 2001.

[5] Ng CY, Chen TP, Ding L, Fung S: Memory characteristics of MOSFETs with densely stacked silicon nanocrystal layers in the gate oxide synthesized by low-energy ion beam. IEEE Trans Electron Device Lett, 27:231, 2006.

[6] Kapetanakis E, Normand P, Tsoukalas D, Beltsios K, Stoemenos J, Zhang S, van der Berg J: Charge storage and interface states effects in Sinanocrystal memory obtained using low-energy Si+ implantation and annealing. Appl.Phys.Lett., 77:3450, 2000.

[7] R. Lankhorst, S. Ketelaars, and M. Wolter. Nature Materials, 4:347, 2005.

[8] S. Tiwari, F. Rana, H. Hanafi, A. Hartstein, E. F. Crabb'e, and K. Chan. Appl.Phys.Lett. 68:1377,1996.

[9] R. Muralidhar, R. F. Steimle, M. Sadd, and R. Rao.IEDM Technical Digest, 2003.

[10] L. J. Guo, E. Leobandung, and Stephen Y. Chou.Science, 275:649, 1997.

[11] S. M. Sze. Modern semiconductor device physics. John Wiley \& Sons,INC, 1998.

[12] Y. Shi, K. Saito, H. Ishikuro, and T. Hiramotob. J. Appl. Phys., 84:2358, 1998.

[13] S. Tiwari, J. A. Wahl, H. Silva, F. Rana, and J. J. Welser. Appl. Phys. A, 71:403, 2000.

[14] Y. Yu and M. Cardona. Fundamentals of semiconductors: physics and materials properties. Spring Press, Berlin, 1996.

[15] A.J. Steckl, J.Xu, H.C. Mogul and S.M. Prokes, J. Elecrochem. Soc, 142(5), pp. 69-71, 1995. 